\title{
Large Dataset Generation, Integration and Simulation in Materials Science, Part II
}

\author{
Jeff Simmons and J.-C. Zhao
}

This is Part II in the series on Large Datasets in Materials Science. Part I appeared in the March issue of JOM and was introduced by the following paragraph:

"As new and rapid materials characterization tools become widely available, large datasets are being generated at a faster and faster pace, which is both exciting and challenging. The exciting aspects are that large amounts of composition, structure, and spectral information about materials are being gathered routinely. This promises to accelerate the traditional scientific pursuits in materials science of establishing processing-structure and structure-property relationships and hypothesis testing. Even more exciting is the promise of quantitative validation of complex physics-based models with the statistically significant datasets that are now becoming possible. The main challenges presented by the emergence of these techniques are rapid acquisition of larger and larger datasets, using this information to provide predictive capabilities in materials systems, and analysis of the increasing volumes of data." The selected articles highlighted here provide a snapshot of some current activities in this expanding field, but in no way attempt to cover the entire field. Such an attempt would be well beyond the page limit of a JOM issue. In fact, this topic, due to the substantial number of materials to cover, has been spread over two months: March and July.

In Part I, we highlighted the modern data generation techniques, relations of structure and materials properties, and advances in interpretation that are enabled by the large datasets that are now becoming available. The articles by Uchic et al., Barabash et al., and Zhao et al. address these in detail. We also highlighted advances in predictions and explanation of materials properties with articles by Lewis et al. and McDowell et al. Finally, Rowenhorst and Lewis described converting image data to knowledge meaningful in a materials context.

The current issue presents the balance of articles in this series and addresses algorithmic advances necessary for analysis of image-based datasets whose size precludes hand analysis. These developments are mainly driven by similar requirements in fields such as biology, homeland security, surveillance, or medical imaging. The key element in the modern approaches is the inclusion of knowledge as to what would constitute a reasonable result of analysis. Materials science, with its wealth of understanding of the origin of microstructure and models of microstructure development, seems to be uniquely positioned to take advantage of, and further develop, these methods.

Multi-spectral and hyper-spectral data is becoming widely used in other fields such as astronomy, and have been a very active area of development in the $\mathrm{x}$-ray community. The article by Kotula and Sorensen describe this technique from data collection to the algorithms developed for data analysis.

The article by Ontman et al. describes the active contour method, based on the method of level sets. With this method, regions of interest are isolated by initially placed boundaries that evolve to conform to those in the image. The equations of motion are constructed to produce reasonable results for the system at hand.

The remaining techniques covered in this issue are addressed by researchers in the signal processing field, who have played key roles in advancing those techniques. The article by Wang et al. describes "Graph-Cut" methods, which allow for incorporation of our extensive fundamental knowledge of interface geometries and their origins. The article by Pollak and Huffman describes "Inverse Diffusion" methods that are analogous to classical diffusion, except that they preserve detail at the boundaries between regions. Finally, Bayesian segmentation techniques show great promise of incorporating materials knowledge, both through models of image formation and through models of microstructure formation. The article by Comer et al. describes Bayesian methods that are immediately applicable to microscope and tomographic data in materials science.

These methods, along with the techniques described in Part I, constitute a necessarily incomplete coverage of advancements made possible by the convergence of computer and information technologies with characterization, analysis, and simulation techniques. This is a nowhere near mature area in materials science and promises many exciting advances in the future.

Jeff Simmons is with the Air Force Research Laboratory and is a member and former chairman of the Advanced Characterization, Testing and Simulation (ACTS) Committee of the Structural Materials Division (SMD) of TMS. J.-C. Zhao, professor, is with the Department of Materials Science and Engineering, The Ohio State University and is the advisor to JOM from the ACTS Committee of SMD.

\section{Jeff Simmons and J.-C. Zhao are TMS Members!}

To read more about them, turn to page 8. To join TMS, visit www.tms.org/Society/Membership.aspx. 